\title{
Inhibition of miR-92a Suppresses Oxidative Stress and Improves Endothelial Function by Upregulating Heme Oxygenase-1 in $d b / d b$ Mice
}

\author{
Lingshan Gou,, ${ }^{1,2}$ Lei Zhao,, ${ }^{1,2}$ Wencong Song,,2 Li Wang, ${ }^{1,2}$ Jian Liu, ${ }^{1,2}$ Hongsong Zhang,,2 \\ Yuhong Huang, ${ }^{1,2}$ Chi Wai Lau, ${ }^{1,2}$ Xiaoqiang Yao, ${ }^{1,2}$ Xiao Yu Tian,, ${ }^{1,2}$ Wing Tak Wong, \\ Jiang-Yun Luo, ${ }^{1,2}$ and Yu Huang ${ }^{1,2}$
}

\section{Abstract}

Aims: Inhibition of microRNA-92a (miR-92a) is reported to suppress endothelial inflammation and delay atherogenesis. We hypothesize that miR-92a inhibition protects endothelial function through suppressing oxidative stress in diabetic $d b / d b$ mice.

Results: In this study, we found elevated expression of miR-92a in aortic endothelium from $\mathrm{db} / \mathrm{d} b$ mice and in renal arteries from diabetic subjects. Endothelial cells (ECs) exposed to advanced glycation end products (AGEs) and oxidized low-density lipoprotein express higher level of miR-92a. Overexpression of miR-92a impairs endotheliumdependent relaxations (EDRs) in C57BL/6 mouse aortas. Overexpression of miR-92a suppresses expression of heme oxygenase-1 (HO-1), a critical cytoprotective enzyme, whereas inhibition of miR-92a increases HO-1 expression in human umbilical vein ECs (HUVECs) and $d b / d b$ mouse aortas. Importantly, miR-92a inhibition by Ad-anti-miR92a improved EDRs and reduced reactive oxygen species (ROS) production in $d b / d b$ mouse aortas. HO-1 inhibition by SnMP or HO-1 knockdown by shHO-1 reversed the suppressive effect of miR-92a inhibition on ROS production induced by AGE treatment in C57BL/6 mouse aortas. In addition, SnMP reversed miR-92a inhibition-induced improvement of EDRs in AGE-treated C57BL/6 mouse aortas and in $d b / d b$ mouse aortas.

Innovation: Expression of miR-92a is increased in diabetic aortic endothelium and inhibition of miR-92a exerts vasoprotective effect in diabetic mice through HO-1 upregulation in ECs.

Conclusion: MiR-92a expression is elevated in diabetic ECs. MiR-92a overexpression impairs endothelial function and suppresses HO-1 expression in ECs. Inhibition of miR-92a attenuates oxidative stress and improves endothelial function through enhancing HO-1 expression and activity in $d b / d b$ mouse aortas. Antioxid. Redox Signal. 28, 358-370.

Keywords: miR-92a, HO-1, endothelial function, diabetes, oxidative stress

\section{Introduction}

D IABETES INCREASES THE risk of developing cardiovascular diseases, such as hypertension and atherosclerosis, through mechanisms involving endothelial dysfunction, which is associated with oxidative stress $(17,34,41)$. Increased ROS production in vascular wall decreases nitric oxide (NO) bioavailability, leading to impaired endothelium-dependent relaxations (EDRs) in diabetes (36). Therefore, approaches ameliorating oxidative stress are regarded as promising strategies to improve endothelial function in diabetes $(31,32,36)$.

\section{Innovation}

In the present study, we provide novel evidence that expression of microRNA-92a (miR-92a) was increased in arteries from diabetic mice and patients. Inhibition of miR-92a protects endothelial function and normalizes reactive oxygen species (ROS) generation through enhancing heme oxygenase-1 (HO-1) expression and activity in $d b / d b$ mice. Thus, targeting miR-92a-HO-1 cascade is effective against diabetic vasculopathy.

\footnotetext{
${ }^{1}$ Institute of Vascular Medicine, Shenzhen Research Institute and Li Ka Shing Institute of Health Sciences, Hong Kong, China.

${ }^{2}$ School of Biomedical Sciences and ${ }^{3}$ School of Life Sciences, Chinese University of Hong Kong, Hong Kong, China.
} 
The miR-17-92 cluster, comprising six individual miRNAs (miR-17, -18a, -19a, -20a, -19b, and -92a), is abundantly expressed in vascular endothelial cells (ECs) (12). Among them, miR-92a plays an important role in modulation of vascular homeostasis $(5,14,50)$. Indirect evidences suggest that miR92a might mediate vascular dysfunction in diabetes. For example, miR-92a was reported to play a proatherogenic role through upregulation of proinflammatory genes in ECs $(7,28$, 49). Another report showed that inhibition of endothelial miR92a attenuates neointimal lesion formation and improves reendothelialization and functional recovery following vascular injury in mice (11). However, direct evidence showing the expression pattern of miR-92a in diabetic endothelium is still lacking, and whether miR-92a regulates oxidative stress and endothelial function in diabetes remains unknown.

Upregulation of the expression and activity of antioxidant enzymes in ECs was demonstrated to be vasoprotective in diabetes $(8,22,39,43)$. For example, HO-1 preserves NO bioavailability to restore endothelial function in diabetic mice (27) although how HO-1 expression is regulated in ECs is incompletely understood. Importantly, a genetic study in type 2 diabetic patients showed that length polymorphism in HO-1 gene promoter in vascular cells is associated with ox- idative stress and higher incidence of coronary artery disease (6). In the present study, we hypothesize that miR-92a inhibition attenuates oxidative stress through upregulation of antioxidant gene expression in diabetic mouse vasculature.

\section{Results}

Oxidative stress contributed to miR-92a upregulation

in diabetic mouse aortas and in ECs exposed

to advanced glycation end products

and oxidized low-density lipoprotein

Using quantitative polymerase chain reaction (q-PCR) analysis, we observed a higher expression of miR-92a in aortas from $d b / d b$ mice compared with those from $d b / m^{+}$mice (Fig. 1A), while no difference was detected in aortas with endothelium removed (Fig. 1B). Removal of endothelium was confirmed by significant loss of mRNA levels of EC-specific markers Cdh5, Pecaml, and $V w f$ (Fig. 1C and Supplementary Fig. S1C, D; Supplementary Data are available online at www.liebertpub.com/ars). We also detected that the expression of other members from the miR-17-92 cluster and the expression pattern of miR-17, $-18 \mathrm{a},-19 \mathrm{a}$, and $-20 \mathrm{a}$ in aortas from $d b / d b$ mice are similar to miR-92a (Supplementary Fig.
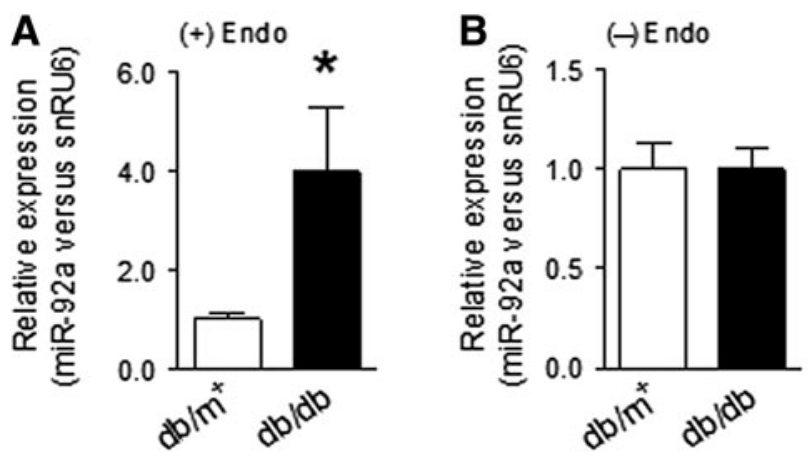

E

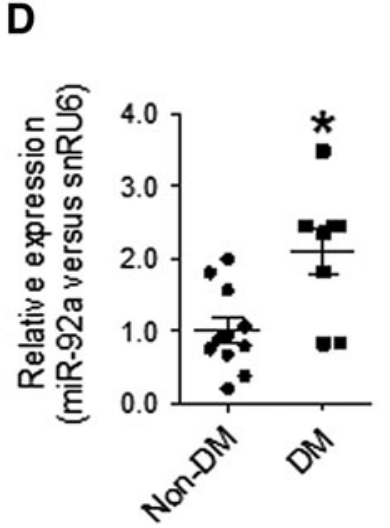

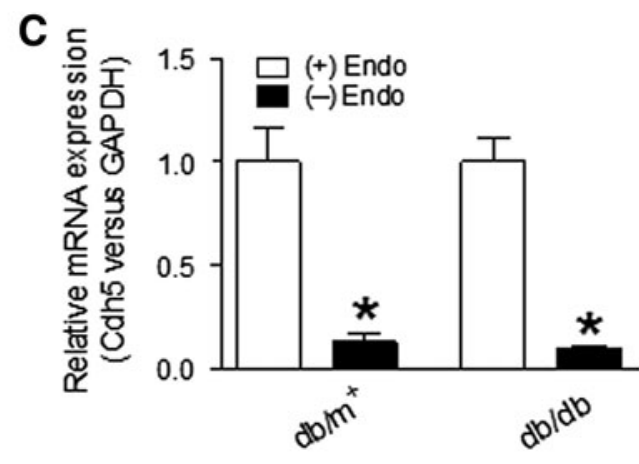

$\mathbf{F}$

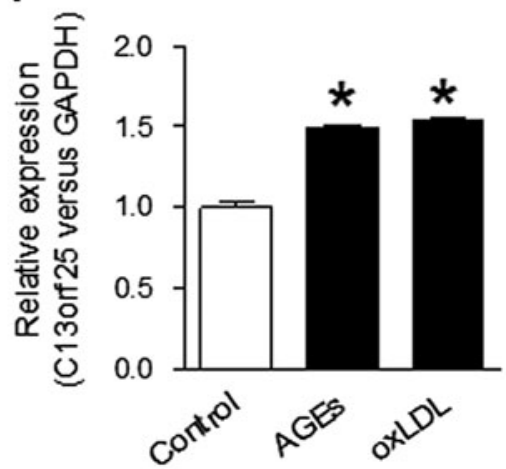

FIG. 1. Elevation of miR-92a expression in diabetic endothelial cells. (A) Increased expression of miR-92a in $d b / d b$ mouse aortas with endothelium (+) Endo. $n=6,{ }^{*} p<0.05$ versus $d b / m^{+}$mice. (B) No elevation of miR-92a expression in $d b / d b$ mouse aortas without endothelium (-) Endo. $n=6,{ }^{*} p<0.05$ versus $d b / m^{+}$mice. (C) Reduced Cdh5 mRNA expression in mouse aortas without endothelium compared with that with endothelium. $n=6, * p<0.05$ versus (+) Endo. (D) Quantitative PCR analysis showing higher expression of miR-92a in renal arteries from diabetic patients compared with nondiabetic subjects. $n=8-11,{ }^{*} p<0.05$ versus non-DM. Data are mean \pm SEM. (E) Treatment with AGEs and oxLDL increased the expression of miR-92a in HUVECs detected by quantitative PCR. $n=5-6, * p<0.05$ versus control. (F) AGEs and oxLDL treatment increased the expression of preliminary transcript of miR-17-92 (C13orf25) in HUVECs detected by quantitative PCR. $n=3$, ${ }^{*} p<0.05$ versus control. AGEs, $200 \mu \mathrm{g} / \mathrm{ml}, 24 \mathrm{~h}$; oxLDL, $100 \mu \mathrm{g} / \mathrm{ml}, 24 \mathrm{~h}$. Data are mean \pm SEM. AGEs, advanced glycation end products; HUVECs, human umbilical vein ECs; oxLDL, oxidized low-density lipoprotein; PCR, polymerase chain reaction; SEM, standard error of mean. 
S1A, B). Importantly, renal arteries from diabetic patients exhibited an increased expression of miR-92a compared with those from nondiabetic subjects (Fig. 1D, Table 1).

In addition, human umbilical vein ECs (HUVECs) treated with advanced glycation end products (AGEs) and oxidized low-density lipoprotein (oxLDL) also expressed a higher level of miR-92a and the preliminary transcript of miR-17-92 cluster (Fig. 1E, F). These results suggest that diabetic condition might increase the expression of miR-92a derived from miR-17-92 cluster in ECs. Next, we tested whether oxidative stress contributed to upregulation of miR-92a in ECs. Tempol is a superoxide dismutase mimetic limiting the production of ROS (52). Tempol treatment decreased miR-92a expression in aortas isolated from $d b / d b$ mice (Supplementary Fig. S2A). Also, the induction of miR-92a by AGEs and ROS generator HX-XO was attenuated by tempol treatment in HUVECs (Supplementary Fig. S2B, C). These results indicate that attenuation of oxidative stress can normalize miR-92a expression in ECs exposed to AGEs and HX-XO.

\section{MiR-92a targets at $\mathrm{HO}-1$ in ECs}

Imbalanced regulation of antioxidant genes contributes to ROS overgeneration in vascular wall in diabetes. Given that our data show increased expression of miR-92a in diabetic mouse ECs, we hypothesize that miR-92a might regulate expression of antioxidant genes in ECs. To identify the candidates, we determined the expression of several oxidant-related genes in HUVECs transduced with Ad-anti-miR-92a, which inhibits miR-92a expression (Supplementary Fig. S3A, B). We found that HO-1 was upregulated by miR-92a inhibition at the protein level, while others were not changed (Supplementary Fig. S4AC). HUVECs treated with AGEs, which increases miR-92a expression, expressed lower level of HO-1 protein (Supplementary Fig. S4D). Q-PCR and western blotting analysis showed that miR-92a downregulated the mRNA and protein expression of HO-1 in HUVECs (Fig. 2A, B).
Using bioinformatic algorithms, HO-1 was predicted as a putative target of miR-92a (Fig. 2C). Indeed, miR-92a overexpression reduced wild-type (WT) but not mutated HO1 3'UTR activity in HUVECs (Fig. 2C). Therefore, modulation of HO-1 expression by miR-92a is highly likely through a direct $3^{\prime}$ UTR binding mechanism. By contrast, inhibition of miR-92a increased HO-1 mRNA and protein expression in HUVECs in a time-dependent manner (Fig. 3A-C and Supplementary Fig. S5). In addition, AGEinduced downregulation of HO-1 mRNA and protein was reversed by miR-92a inhibition (Fig. 3D, E). Taken together, our results demonstrate that $\mathrm{HO}-1$ is a previously unrecognized target of miR-92a in ECs.

To determine whether HO-1 expression is downregulated in endothelium of $d b / d b$ mice, we performed en face fluorescence staining of HO-1 in mouse aortas. The results showed that HO1 level was decreased in aortic endothelium from $d b / d b$ mice, compared with that from $\mathrm{db} / \mathrm{m}^{+}$mice (Fig. 3F). In addition, HO-1 mRNA level was elevated in aortas without endothelium from $d b / d b$ mice compared with that from $d b / m^{+}$mice (Supplementary Fig. S4F). These data suggest that elevated miR92a is likely to suppress HO-1 expression in vascular ECs in $d b / d b$ mice.

\section{Overexpression of miR-92a impairs endothelial function}

To test whether the elevated expression of miR-92a plays a functional role in ECs, we overexpressed miR-92a in mouse aortas using adeno-associated virus (AAV) vector. $E x$ vivo transduction of AAV-miR-92a for $36 \mathrm{~h}$ increased miR-92a expression in C57BL/6 mouse aortas (Fig. 4A). MiR-92a overexpression impaired ACh-induced EDRs, without affecting sodium nitroprusside (SNP)-induced endothelium-independent relaxations (EIRs) in C57BL/6 mouse aortas (Fig. 4B, C). These results indicate that increased miR-92a level in $d b / d b$ mouse aortic endothelium impairs endothelial function.

Table 1. Clinical Parameters of Patients

\begin{tabular}{|c|c|c|c|c|c|}
\hline $\begin{array}{l}\text { Patient } \\
\text { no. }\end{array}$ & $\operatorname{Sex}$ & Age & $\begin{array}{c}D M / \\
\text { non-DM }\end{array}$ & Nephrectomy information & $\begin{array}{l}\text { Valid endocrine } \\
\text { anthropometric parameter }\end{array}$ \\
\hline 1 & M & 67 & Y & Left radical nephrectomy & BW: $80, \mathrm{HbA1c:} 5.9 \%, \mathrm{Cr}: 812$ \\
\hline 2 & M & 67 & $\mathrm{Y}$ & Laparoscopic right radical nephrectomy & BW: 74, HbA1c: $6.1 \%$, Cr: 157 \\
\hline 3 & $\mathrm{~F}$ & 63 & $\mathrm{Y}$ & Left nephroureterectomy & BW: $65, \mathrm{HbA} 1 \mathrm{c}: 6.0 \%, \mathrm{Cr}: 119$ \\
\hline 4 & M & 66 & Y & $\begin{array}{l}\text { Retroperitoneoscopic right radical } \\
\text { nephrectomy }\end{array}$ & BW: $58.2, \mathrm{Cr}: 81$ \\
\hline 5 & M & 67 & $\mathrm{Y}$ & & BW: 83.6, Cr:118 \\
\hline 6 & $\mathrm{~F}$ & 65 & $\mathrm{Y}$ & & BW: $50.3, \mathrm{Cr}: 66$ \\
\hline 7 & $\mathrm{~F}$ & 74 & $\mathrm{Y}$ & Nonfunction right kidney & BW: 51.9, Cr:128 \\
\hline 8 & $\mathrm{~F}$ & 66 & $\mathrm{Y}$ & & BW: $63.5, \mathrm{HbA} 1 \mathrm{c}: 7.4 \%, \mathrm{Cr}: 51$ \\
\hline 9 & $\mathrm{~F}$ & 62 & $\mathrm{~N}$ & Left radical nephrectomy & BW: 48, Cr: 95 \\
\hline 10 & M & 53 & $\mathrm{~N}$ & & BW: 77.8, Cr: 98 \\
\hline 11 & $\mathrm{~F}$ & 60 & $\mathrm{~N}$ & & BW: 45, HbA1c: $6.0 \%$, Cr: 57 \\
\hline 12 & $\mathrm{~F}$ & 78 & $\mathrm{~N}$ & Renal transitional cell carcinoma & BW: $55.8, \mathrm{Cr}: 112$ \\
\hline 13 & $\mathrm{~F}$ & 68 & $\mathrm{~N}$ & Renal tumor & BW: 55.7, Cr: 121 \\
\hline 14 & M & 58 & $\mathrm{~N}$ & & BW: 56.3 , HbAlc: $6.4 \%$, Cr: 75 \\
\hline 15 & $\mathrm{~F}$ & 69 & $\mathrm{~N}$ & & BW: $60, \mathrm{Cr}: 74$ \\
\hline 16 & M & 58 & $\mathrm{~N}$ & & BW: 79, Cr: 120 \\
\hline 17 & $\mathrm{~F}$ & 79 & $\mathrm{~N}$ & & BW: $51.2, \mathrm{HbA} 1 \mathrm{c}: 5.5 \%, \mathrm{Cr}: 83$ \\
\hline 18 & M & 60 & $\mathrm{~N}$ & & \\
\hline 19 & M & 58 & $\mathrm{~N}$ & & Cr: 118 \\
\hline
\end{tabular}

BW, body weight, $\mathrm{kg}$; Cr, creatinine, $\mu \mathrm{mol} / \mathrm{L}$; DM, diabetes; HbAlc, glycated hemoglobin; non-DM, without diabetes. 
FIG. 2. miR-92a inhibits HO-1 expression in HUVECs. (A) AAV-miR-92a transduction downregulated HO-1 mRNA level in HUVECs. $n=6, * p<0.05$ versus vector. (B) AAV-miR-92a transduction decreased HO-1 protein expression in $\mathrm{HU}$ VECs. $n=4, * p<0.05$ versus vector. (C) Dual luciferase assay showing WT but not mutant HO-1 3'UTR activity was suppressed in HUVECs transfected with pAAV-miR92a. $n=5, * p<0.05$ versus pAAV-vector + Luc-HO-1 (WT); ${ }^{\#} p<0.05$ versus $\mathrm{pAAV}$ miR-92a + Luc-HO-1 (WT). Data are mean \pm SEM. Vector, AAV-vector; miR-92a, AAVmiR-92a; AAV, adenoassociated virus; HO-1, heme oxygenase-1; WT, wild type.

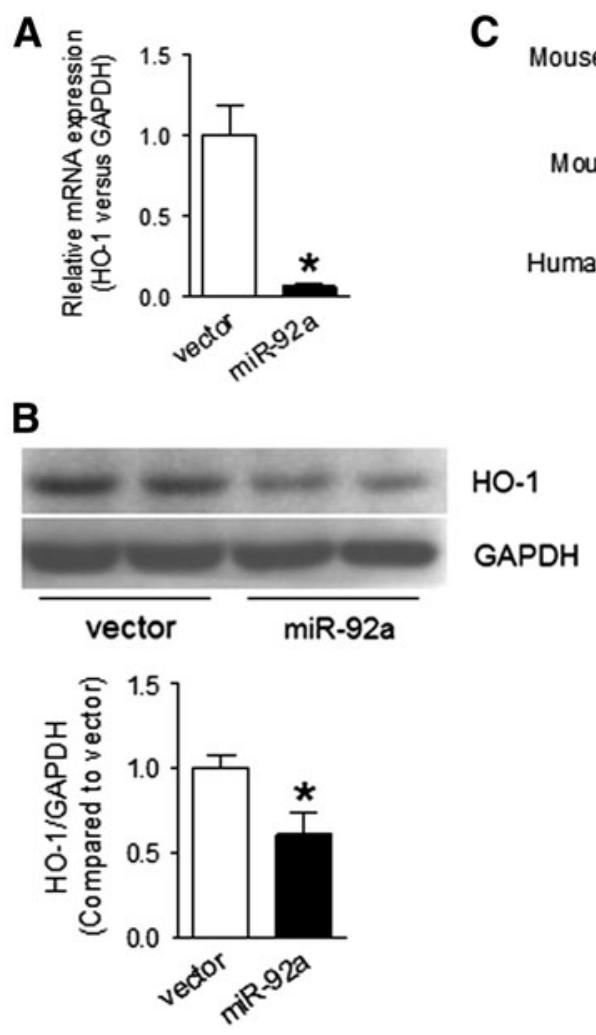

Mouse HO-1 3'UTR 5'...CUCUAACUUCUGUGUGAAAUA... miR-92a 3'...GUCCGGCCCU'GU'UCÁl IIIJII.

Mouse HO-1 3'UTR 5'... UUAUGCCAUGUUUUUGCAAUA... miR-92a 3'... GUCCGGCCCÚGUUCACGUUAU.. uman HO-1 3'UTR 5'... CUGG CUCAGCCUCAAAUGCAGUA.. I I I ||| ||||||| miR-92a 3'...UGUCCGGCCCUGUUCACGUUAU...

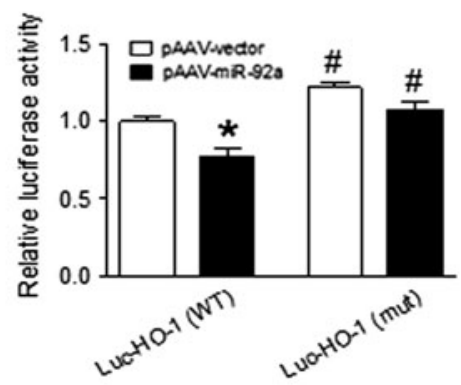

To determine the role of HO- 1 in miR-92a overexpressioninduced impairment of EDRs, aortic rings were treated with HO-1 inducer hemin to activate HO-1 before AAVtransduction of miR-92a. Detection of gene expression by q-PCR showed that miR-92a overexpression-induced downregulation of HO-1 mRNA level was increased by hemin treatment in mouse aortic rings (Fig. 4D). Hemin treatment also reversed the impairment of EDRs in aortas treated with AAV-miR-92a and AGEs (Fig. 4E, F). These data indicate that HO-1 activation by hemin is vasoprotective to antagonize the harmful effect of miR-92a overexpression.

\section{Inhibition of miR-92a suppresses oxidative stress and improves ACh-induced EDRs in diabetic mice}

Increased oxidative stress contributes to endothelial dysfunction in diabetes $(17,34)$. Based on our finding that the cytoprotective antioxidant enzyme HO-1 is inhibited by miR92a overexpression, we hypothesize that miR-92a-inhibitioninduced HO-1 upregulation might suppress ROS production in diabetic mouse ECs. First, we injected Ad-anti-miR-92a into $\mathrm{db} /$ $d b$ mice to inhibit miR-92a. The ROS level indicated by dihydroethidium (DHE) staining in aortic endothelium from $d b / d b$ mice was higher compared with that from $d b / m^{+}$mice (Fig. 5B). Ad-anti-miR-92a delivery reduced miR-92a expression and normalized ROS overproduction in $d b / d b$ mouse aortic endothelium (Fig. 5A-C). Likewise, lucigenin chemiluminescence assay showed that miR-92a inhibition reduced superoxide levels in $d b / d b$ mouse aortas (Fig. 5D). Moreover, comparable to the effect of ROS scavenger tempol, Ad-anti-miR-92a reversed AGE-induced superoxide production in cultured mouse aortas (Supplementary Fig. S6B). In addition, Ad-anti-miR-92a treatment in $d b / d b$ mice also normalized the elevated levels of 3-nitrotyrosine, the oxidative stress biomarker in aortas (Supplementary Fig. S7). Collectively, these results demonstrate that inhibition of miR-92a suppresses ROS overproduction in ECs under diabetic conditions.

To assess whether miR-92a inhibition improves endothelial function in $d b / d b$ mice, we measured ACh-induced EDRs in mouse aortas treated with Ad-anti-miR-92a. In vivo delivery of Ad-anti-miR-92a for 1 week improved EDRs, while SNP-induced EIRs were not affected (Fig. 5E, F). In addition, both endothelial nitric oxide synthases (eNOS) and p-eNOS (S1176) levels in $d b / d b$ mouse aortas were upregulated by miR-92a inhibition (Supplementary Fig. S8A). Moreover, Ad-anti-miR-92a also increased eNOS and p-eNOS protein levels in HUVECs in the presence of AGEs (Supplementary Fig. S8B). On the contrary, miR-92a overexpression decreased levels of both p-eNOS and eNOS in HUVECs (Supplementary Fig. S8C). These results suggest that miR-92a inhibition-induced improvement of EDRs is likely mediated by increased NO bioavailability as reflected by upregulated eNOS and p-eNOS protein in $d b / d b$ mouse aortas.

To confirm this likelihood, we assayed the level of nitrite/ nitrate, the breakdown products of NO, and found that Ad-antimiR-92a treatment increased nitrite/nitrate levels in both the culture medium for HUVECs and the plasma collected from $d b / d b$ mice (Supplementary Fig. S8D, E). These data demonstrate that miR-92a inhibition protects endothelial function through increasing NO bioavailability in $d b / d b$ mice.

We measured HO-1 mRNA and protein level in aortas from $d b / m^{+}$and $d b / d b$ mice treated with adenoviral vectors. Ad-antimiR-92a transduction increased HO-1 mRNA and protein expression in $d b / d b$ mouse aortas (Fig. 5G, H). However, no difference of $\mathrm{HO}-1$ mRNA and protein expression in 


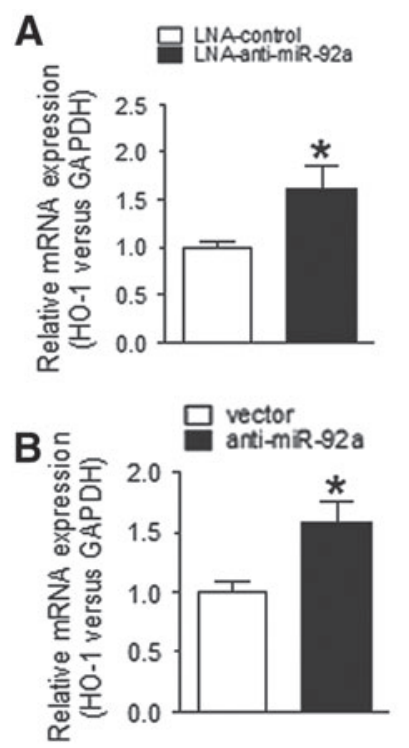

C
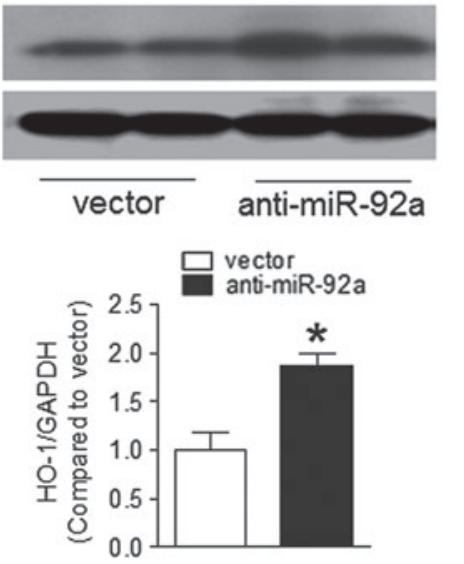

D
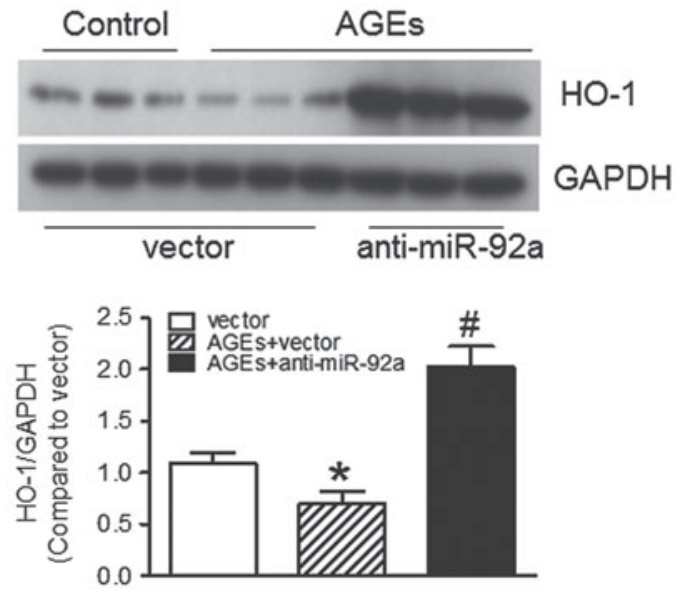

E

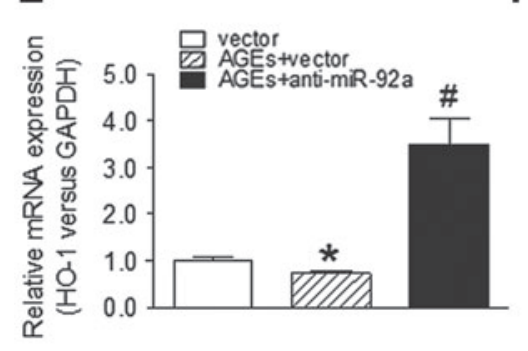

$\mathbf{F}$

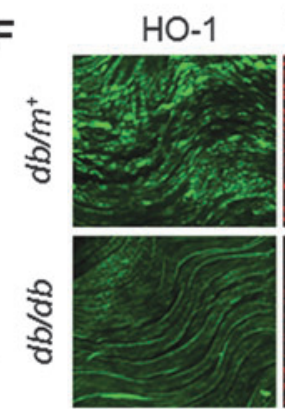

VE-cadherin
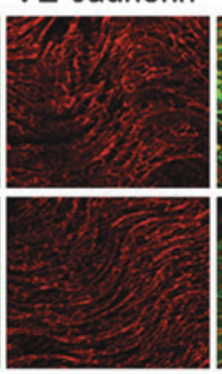

Merge

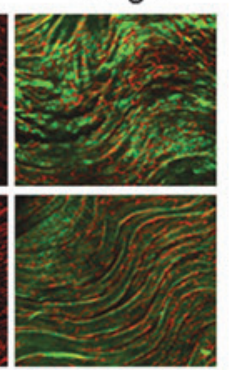

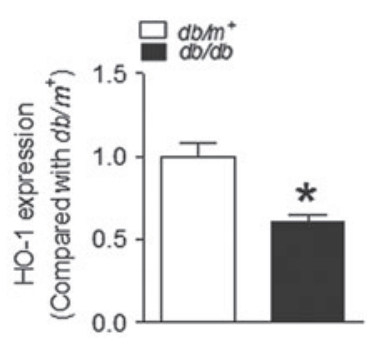

FIG. 3. Inhibition of miR-92a increases HO-1 expression in ECs. (A) LNA-anti-miR-92a transfection increased HO-1 mRNA level in HUVECs. $n=4, * p<0.05$ versus LNA-control. (B) Ad-anti-miR-92a transduction enhanced HO-1 mRNA level in HUVECs. Forty-eight hours after Ad-anti-miR-92a transduction, HUVECs were collected for RNA extraction. $n=8, * p<0.05$ versus vector. (C) Ad-anti-miR-92a transduction on HO-1 protein expression in HUVECs. Forty-eight hours after Ad-anti-miR-92a transduction, HUVECs were harvested for protein extraction. $n=6,{ }^{*} p<0.05$ versus vector. (D) Adanti-miR-92a transduction increased HO-1 protein expression in AGE-treated HUVECs. Forty-eight hours after Ad-antimiR-92a transduction, HUVECs were subjected to AGE treatment for $24 \mathrm{~h} . n=7,{ }^{*} p<0.05$ versus vector; ${ }^{\#} p<0.05$ versus AGEs + vector. (E) Ad-anti-miR-92a transduction increased HO-1 mRNA level in AGE-treated HUVECs. $n=4, * p<0.05$ versus vector; ${ }^{\#} p<0.05$ versus AGEs + vector. (F) Representative images and summarized data showing en face immunofluorescence staining of HO-1 expression in ECs of intact mouse aortae. $n=4, * p<0.05$ versus $\mathrm{db} / \mathrm{m}^{+}$mice. Data are mean \pm SEM. anti-miR-92a, Ad-anti-miR-92a; Vector, Ad-vector.

endothelium-intact aortas was found between $\mathrm{db} / \mathrm{m}^{+}$and $\mathrm{db} / \mathrm{db}$ mice. While the en face imaging results showed a decreased endothelial HO-1 level in $d b / d b$ mice (Fig. 3F). It is likely that the increased expression of HO-1 in aortic smooth muscle cells may offset the reduction of $\mathrm{HO}-1$ expression in diabetic aortic ECs when the aortas were assayed for HO-1 levels using q-PCR and western blot (Supplementary Fig. S4F).

\section{HO-1 inhibition by SnMP reversed the effect of miR-92a inhibition on AGE-induced ROS production in C57BL/6 mouse aortas and EDRs in $\mathrm{db} / \mathrm{db}$ mouse aortas}

To determine whether HO-1 mediates the effect of miR$92 \mathrm{a}$ inhibition on attenuation of oxidative stress and improvement of EDRs in $d b / d b$ mouse aortas, we used SnMP $(30 \mu M)$ to inhibit HO-1 activity as indicated by changes in bilirubin levels in HUVECs (Supplementary Fig. S9A, B). As shown by en face DHE staining, the effect of ex vivo transduction of Ad-anti-miR-92a on AGE-induced ROS production in C57BL/6 mouse aortas was reversed by SnMP, while the expression of HO-1 was not affected (Fig. 6A-D). Consistently, miR-92a inhibition by LNA-anti-miR-92a transfection attenuated AGE-induced ROS production in HUVECs (Fig. 6E, F).

To exclude the possible off-target effect of SnMP, AAVshHO-1, which specifically knocks down HO-1 expression, was used to confirm that HO-1 silencing also reverses the effect of miR-92a-inhibition on ROS production. The results of en face DHE staining showed that shHO-1 reversed the suppressive effect of anti-miR-92a on AGE-induced ROS production (Supplementary Fig. S10A, B). Functionally, SnMP treatment abolished miR-92a inhibition-induced improvement of EDRs in AGE-treated C57BL/6 mouse aortas, with no effect on tempol-rescued EDRs (Fig. 7A-D). Furthermore, administration of SnMP to $d b / d b$ mice for 1 week reversed the protective effect of miR-92a inhibition on EDRs in $d b / d b$ mouse aortas, and SNP-induced EIRs were not 


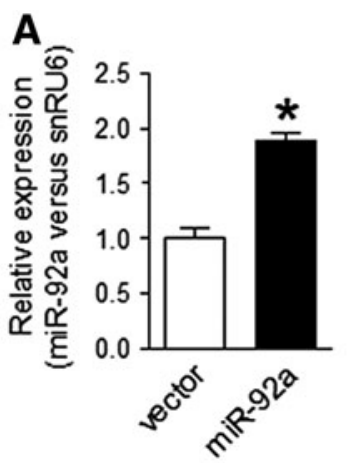

B

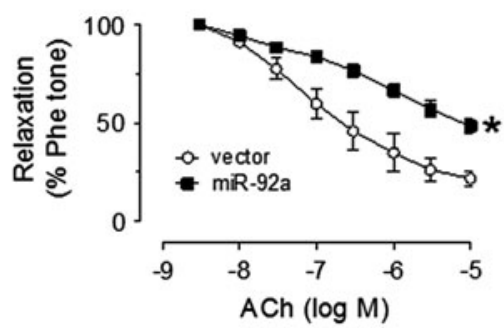

E

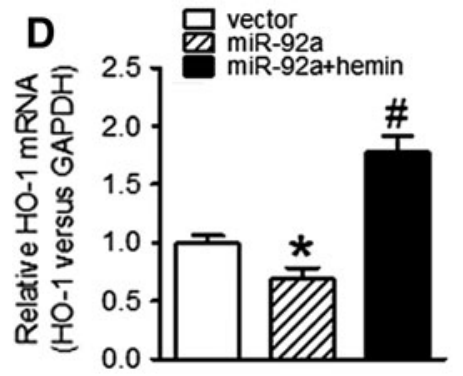

$B$

C

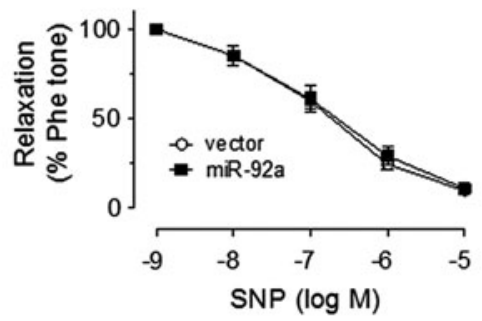

$\mathbf{F}$

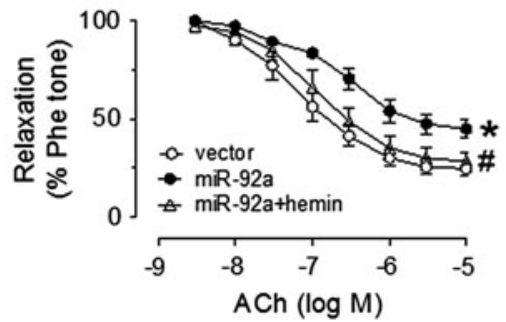

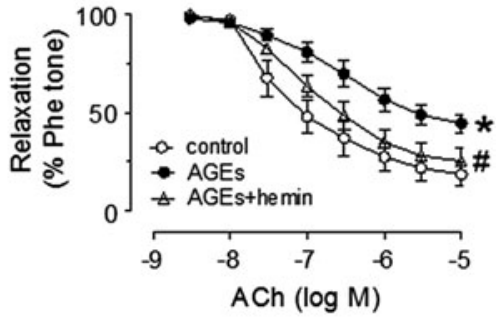

FIG. 4. Overexpression of miR-92a impairs endothelium-dependent relaxations (EDRs) in mouse aortas. (A) $E x$ vivo AAV-miR-92a transduction increased miR-92a expression in mouse aortas from C57BL/6 mice. $n=3,{ }^{*} p<0.05$ versus vector. (B) AAV-miR-92a impaired EDRs (C), while it did not affect SNP-induced relaxations in mouse aortas from C57BL/6 mice. $n=5,{ }^{*} p<0.05$ versus vector. (D) AAV-miR-92a transduction decreased miR-92a expression, while hemin increased HO- 1 mRNA level in cultured mouse aortas. $n=3-4,{ }^{*} p<0.05$ versus vector; ${ }^{\#} p<0.05$ versus miR-92a. (E) Hemin treatment improved AAV-miR-92a transduction-induced impairment of EDRs in mouse aortas. $n=4$, ${ }^{*} p<0.05$ versus vector; ${ }^{\#} p<0.05$ versus miR-92a. (F) Hemin treatment improved AGE-induced impairment of EDRs in mouse aortas. $n=4,{ }^{*} p<0.05$ versus control; ${ }^{\#} p<0.05$ versus AGEs. Data are mean \pm SEM. miR-92a, AAV-miR-92a; SNP, sodium nitroprusside; Vector, AAV-vector.

affected (Fig. 7E, F). Collectively, these results demonstrate that HO-1 mediates the vasoprotective effect of miR-92a inhibition in $d b / d b$ mouse ECs.

\section{Discussion}

MiRNAs play important roles in regulating cardiovascular diseases through targeting a broad network of related genes $(3,9,33)$. Increased expression of endothelial miR-92a is involved in the atherosclerotic progression $(7,28)$. In the present study, we found an increased miR-92a expression in $d b / d b$ mouse aortic ECs (MAECs) and in renal arteries from diabetic patients. Our data showed that the upregulated miR92a in diabetic mouse ECs is possibly oxidative stress dependent, and miR-92a inhibition normalized ROS level in vascular wall and improved EDRs in diabetic $d b / d b$ mouse aortas.

Mechanistically, miR-92a targets at endothelial HO-1, and the upregulated HO-1 mediates miR-92a-inhibition-induced beneficial effect on endothelial function in $d b / d b$ mice. The results highlight the potential importance of miR-92a in mediation of vascular dysfunction in diabetes, and normalization of the upregulated miR-92a level might hold therapeutic promise against diabetic vasculopathy.

One mechanism responsible for the upregulation of miRNA expression under diabetic condition is oxidative stress. For example, ROS-dependent elevation of miR-200c in ECs increases COX-2 expression to impair endothelial function in diabetic mice (54). Existing evidences show that miR-92a is abundantly expressed in ECs and exhibits antiangiogenic function (12). Oxidative stress-dependent activation of sterol regulatory element binding protein 2 (SREBP2) is reported to transactivate miR-92a expression in ECs (7). Despite the importance of miR-92a in regulation of vascular health, the expression pattern and how miR-92a expression is regulated in diabetic mouse ECs remain unclear. Our results showed that diabetes promotes miR-92a expression, which is likely mediated by increased oxidative stress in vascular wall of diabetic mice. The results were supported by the observation that tempol treatment normalized the increased miR-92a expression in $d b / d b$ mouse aortas and in HUVECs treated with AGEs and HX-XO.

Dyslipidemia, mainly characterized with elevation of circulating cholesterol and triglyceride levels, is recognized as a major risk factor for diabetic complications $(20,44)$. In addition, increased AGE formation contributed to endothelial dysfunction in diabetes $(4,42)$. Both oxLDL and AGEs are able to trigger oxidative stress in ECs $(16,18)$. These previous findings suggest that lipoprotein- and AGE-associated oxidative stress is highly possible to mediate the upregulation of miR-92a in diabetic ECs. Further experiment using tempol treatment in $d b / d b$ mice is needed to test this hypothesis. Moreover, diabetes increases SREBP2, which is a transcriptional activator of miR-92a in renal tissues (21). Therefore, whether the increased miR-92a expression in diabetic ECs is mediated by SREBP2 is worthy of further investigation. In addition, upregulation of nuclear factor- $\kappa \mathrm{B}(\mathrm{NF}-\kappa \mathrm{B})$ activity increases miR-92a expression in ECs (7). However, in our 
A

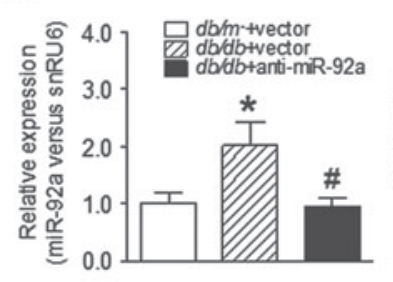

E

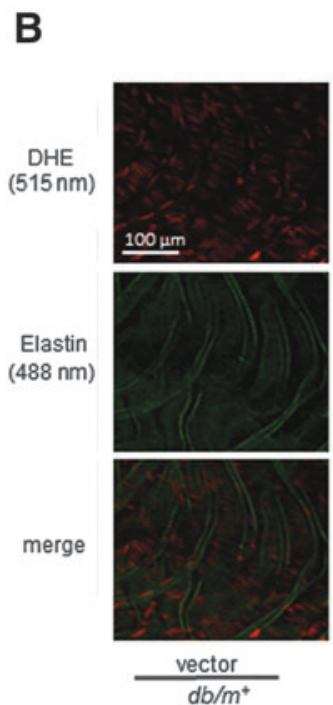

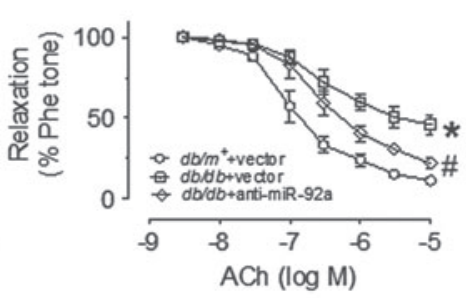

F

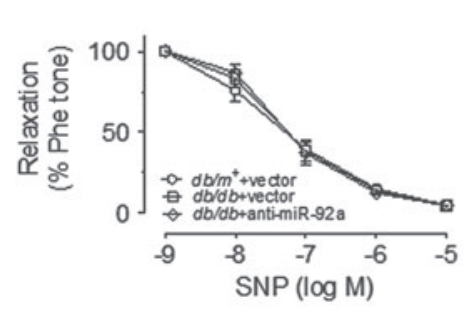

G

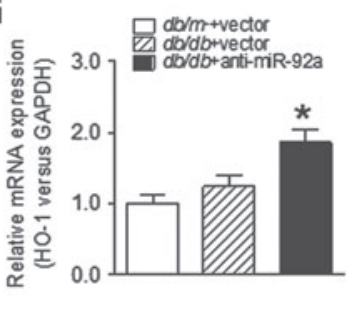

C
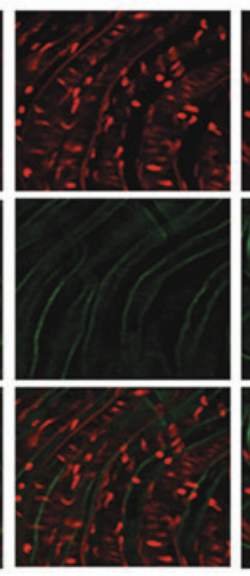

vector
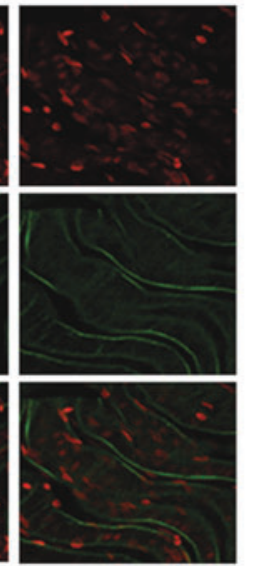

anti-miR-92a

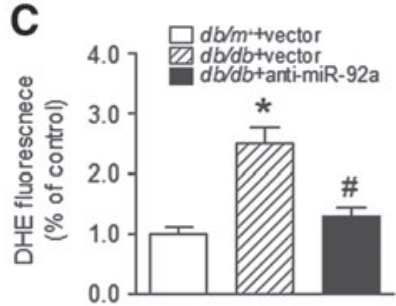

H
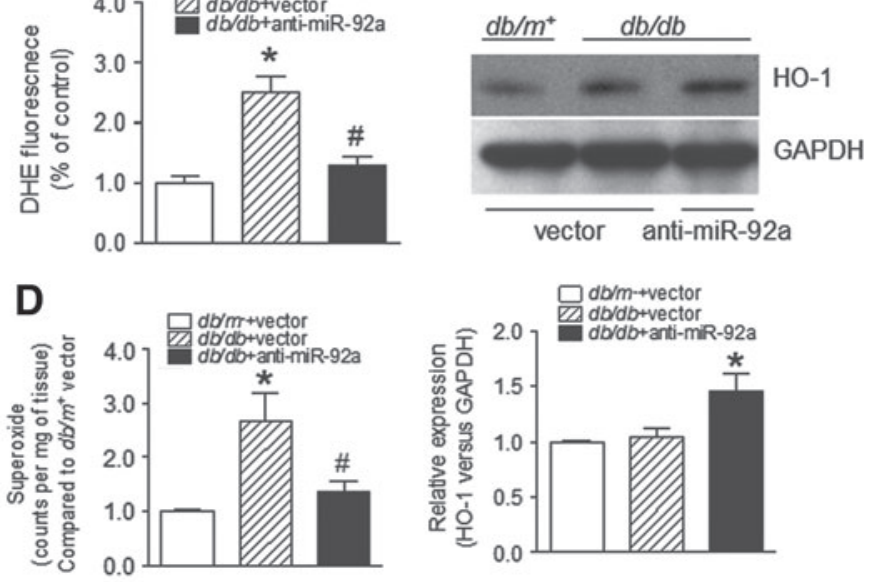

FIG. 5. Inhibition of miR-92a suppresses oxidative stress and improves ACh-induced EDRs in diabetic endothelium. (A) Delivery of Ad-anti-miR-92a reduced miR-92a expression in $d b / d b$ mouse aortas detected by quantitative PCR. $n=5-6$, * $p<0.05$ versus $d b / m^{+}+$vector; ${ }^{\#} p<0.05$ versus $d b / d b+$ vector. Representative images (B) and summarized data (C) showing ROS levels indicated by dihydroethidium (DHE, $5 \mu M$ ) staining in en face endothelium of aortas from $d b / m^{+}$ and $d b / d b$ mice treated with vector or anti-miR-92a. (D) Lucigenin-enhanced chemiluminescence assay showing Ad-antimiR-92a reduced ROS production in $d b / d b$ mouse aortas. $n=4,{ }^{*} p<0.05$ versus $d b / m^{+}+$vector; ${ }^{\#} p<0.05$ versus $d b / d b+$ vector. Ad-anti-miR-92a improved EDRs (E), while it did not affect SNP-induced relaxations (F) in mouse aortas from $d b / d b$ mice. $n=5, * p<0.05$ versus $d b / m^{+}+$vector; ${ }^{*} p<0.05$ versus $d b / d b+$ vector. Ad-anti-miR-92a increased HO- $1 \mathrm{mRNA}$ (G) and HO-1 protein $(\mathbf{H})$ level in mouse aortas from $d b / d b$ mice. $n=5$, ${ }^{*} p<0.05$ versus $d b / m^{+}+$vector; ${ }^{*} p<0.05$ versus $d b / d b+$ vector. Data are mean \pm SEM. anti-miR-92a, Ad-anti-miR-92a; ROS, reactive oxygen species; Vector, Ad-vector.

preliminary test, IL-1 $\beta$ treatment failed to increase miR-92a expression in HUVECs (Supplementary Fig. S2D). Although $\mathrm{NF}-\kappa \mathrm{B}$ is known to be activated in diabetic vasculature, it is less likely to account for miR-92a elevation in diabetes based on our preliminary results.

Recently, miRNAs are recognized as important regulators of multiple cellular processes, including oxidative stress (51). In diabetic cardiomyocytes, miR-144 overexpression increases ROS production, while miR-144 inhibition abates excessive ROS levels (53). Furthermore, downregulation of miR-137 reduces high glucose-induced cellular oxidative stress in ECs (25). In contrast, augmenting the expression of miR-200c in HUVECs leads to increased oxidative stress and cellular injury (29). These reports highlight miRNAs as critical regulators of cellular ROS production, which can be targeted by miRNA inhibitors or activators. In this study, our results revealed that miR-92a inhibition reduces endothelial oxidative stress in vivo and in vitro. To our knowledge, this is the first report describing the functional significance of miR92a inhibition in combating against oxidative stress in diabetes. In addition, our findings further support the notion that
miRNAs are functional regulators of oxidative stress in pathological conditions.

ROS overproduction critically contributes to diabetic endothelial dysfunction. This adverse effect is likely through the mechanisms involving decreased NO bioavailability and direct damage to ECs $(34,37)$. In contrast, normalization of oxidative stress using antioxidants reduces vascular injury in diabetes, suggesting the beneficial effect of antioxidant treatment on diabetic vasculopathies $(23,47)$. Our results showed that miR$92 \mathrm{a}$ inhibition increased p-eNOS and plasma nitrite/nitrate level and improved EDRs in $d b / d b$ mouse aortas. This suggests that the improvement of endothelial function by inhibition of miR-92a in ECs is most likely through enhancement of eNOSNO signaling in diabetes. MiR-92a inhibition also increased basal eNOS protein levels. However, whether the improvement of EDRs is secondary to the suppressed endothelial ROS levels by miR-92a inhibitor is currently unsolved. Therefore, further experiments using ROS scavengers to treat $d b / d b$ mice before anti-miR-92a administration are needed to determine the relative contribution of ROS suppression and eNOS upregulation to miR-92a inhibition-induced preservation of NO. 

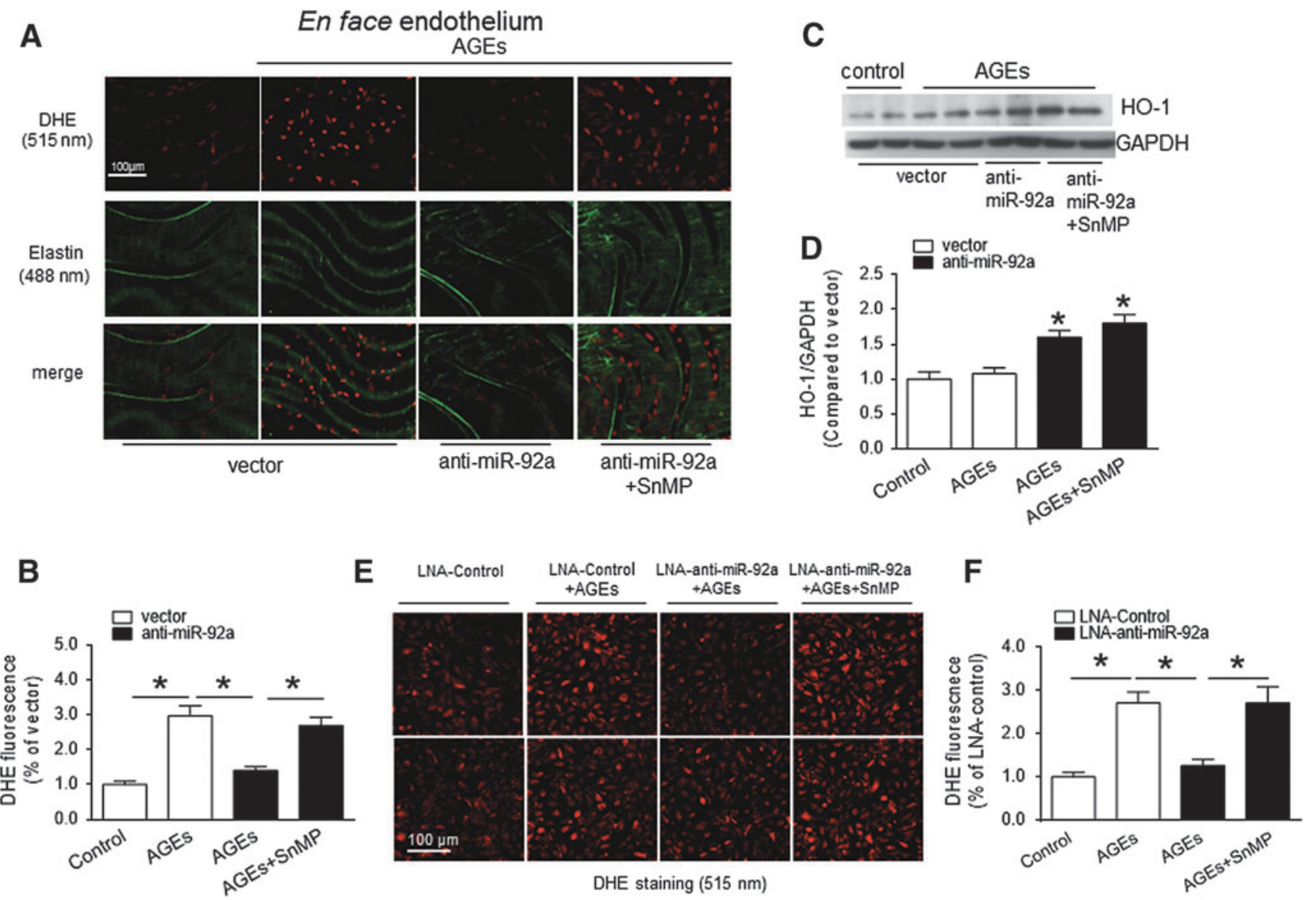

FIG. 6. HO-1 inhibition by SnMP reversed the effect of miR-92a inhibition on ROS production induced by AGE treatment in C57BL/6 mouse aortas. Representative images (A) and summarized data (B) showing HO-1 inhibitor SnMP attenuated the effect of Ad-anti-miR-92a on AGE-induced ROS production indicated by intensity of DHE $(5 \mu M)$ staining in en face endothelium from mouse aortas. $n=4,{ }^{*} p<0.05$ versus respective control. Representative images (C) and summarized data (D) showing Ad-anti-miR-92a increased HO-1 protein expression in mouse aortas treated with AGEs and SnMP. $n=4, * p<0.05$ versus respective control. Representative images (E) and summarized data (F) showing SnMP reversed the effect of LNA-anti-miR-92a on AGE-induced ROS production in HUVECs. $n=5$, * $p<0.05$ versus respective control. Data are mean \pm SEM.

We identified the target genes that might contribute to the protective effect of miR-92a inhibition on diabetic ECs. We performed a screening on expression of oxidant-related enzymes in HUVECs treated with miR-92a inhibitor. MiR-92a inhibition increased HO-1 protein expression, while others were not changed. Dual luciferase assays with WT and mutated 3-'UTR of the HO-1 gene support the prediction that miR-92a inhibition may directly upregulate $\mathrm{HO}-1$ expression in ECs. As a critical inducible antioxidant and cytoprotective enzyme, few studies investigated the mechanisms underlying the regulation of HO-1 expression in ECs, especially under diabetic conditions. In the present study, we found that AGEs downregulated the expression of $\mathrm{HO}-1$, which was increased by miR-92a inhibition in HUVECs. Consistently, the en face staining data showed a downregulation of HO-1 level in aortic ECs from $d b / d b$ mice. Importantly, we also demonstrate that HO-1 mediates the vasoprotective effect of miR92a inhibition in diabetic mouse ECs.

HO-1 is critically involved in regulation of organ function, such as the heart, kidney, and brain $(2,10,19,35)$. Carbon monoxide and bilirubin, two molecules downstream of HO-1 signaling, are the major mediators of the regulatory function of HO-1 in tissue homeostasis and redox signaling $(38,40)$. Recent studies have implicated that $\mathrm{HO}-1$ polymorphisms are associated with a high risk of vascular diseases $(26,30)$. Induction of HO-1 exerts arterial antioxidant actions and causes vascular relaxations $(1,13,27,45)$. We showed that miR-92a inhibition increased $\mathrm{HO}-1$ expression, and $\mathrm{HO}-1$ inhibitor SnMP reversed the beneficial effect of miR-92a inhibition on endothelial function in $d b / d b$ mouse aortas. Induction of krüppel-like factor 2 (KLF2), a target gene of miR-92a, also enhances HO-1 expression in ECs (15). We found that miR-92a inhibition increased KLF2 expression in ECs (Supplementary Fig. S4E), which was consistent with previous reports (50). Therefore, it is also valuable to study whether induction of HO-1 by miR-92a inhibition can be contributed by KLF2 in ECs under diabetic conditions.

In conclusion, our study provides the first line of evidence that increased expression of miR-92a in arteries from diabetic mice and patients. MiR-92a inhibition improves endothelial function and normalizes excessive ROS levels through enhancing HO-1 expression and activity (Fig. 7G). Thus, 
A

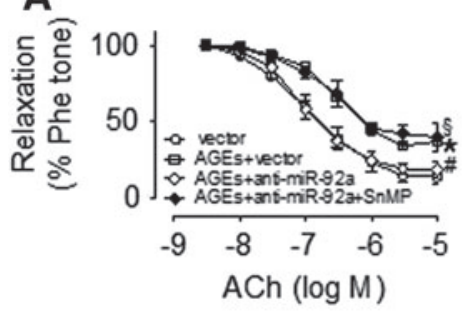

C

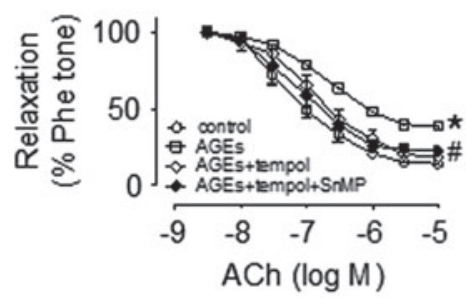

E

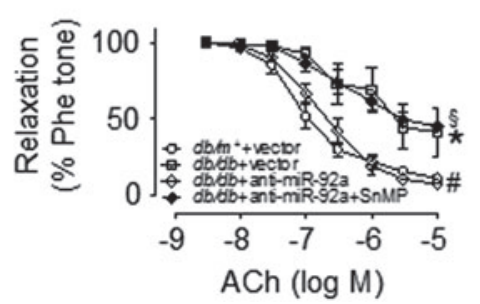

B

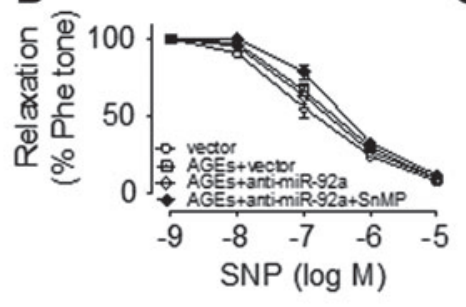

D

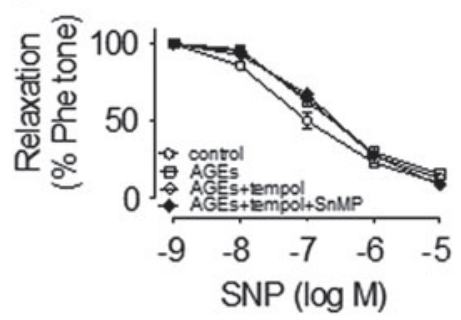

F

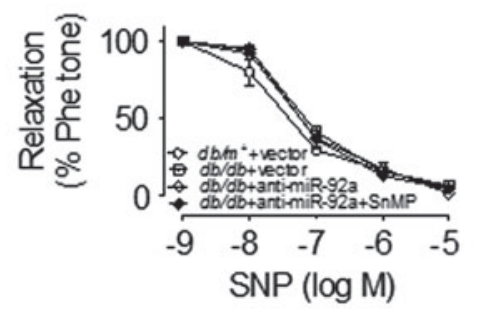

G

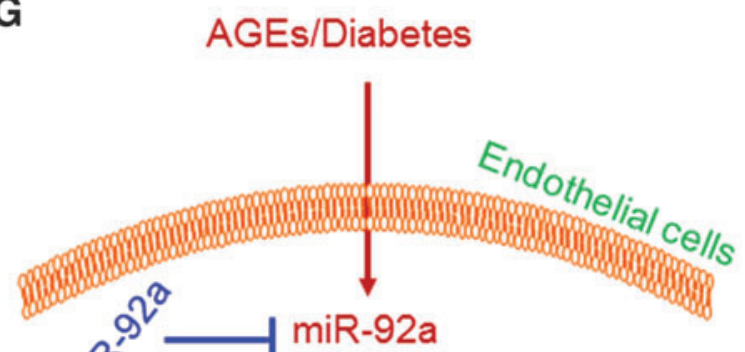

Hemin

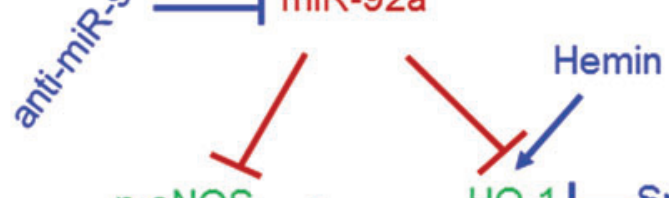

p-eNOS

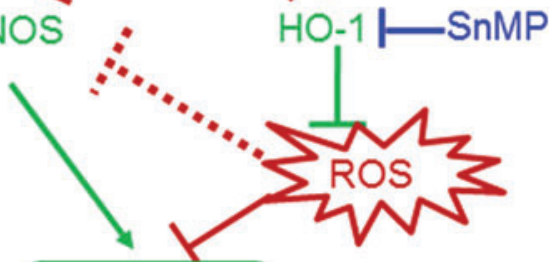

Nitric oxide

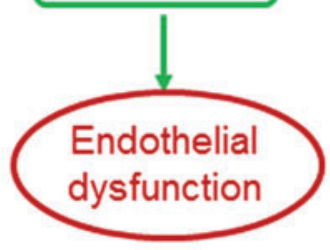

FIG. 7. HO-1 inhibition by SnMP reversed the effect of miR-92a inhibition on EDRs in AGE-treated C57BL/6 mouse aortas and in $\boldsymbol{d} b / \boldsymbol{d} b$ mouse aortas. (A) SnMP reversed the effect of anti-miR-92a-induced improvement of EDRs (B), while it did not affect SNP-induced relaxations in C57BL/7 mouse aortas treated AGEs. $n=4,{ }^{*} p<0.05$ versus vector; ${ }_{p} p<0.05$ versus AGEs + vector; ${ }^{8} p<0.05$ versus AGEs + anti-miR-92a. (C) SnMP did not affect tempol-induced improvement of EDRs and (D) SNP-induced relaxations in C57BL/7 mouse aortas treated with AGEs. $n=4, * p<0.05$ versus control; ${ }^{\#} p<0.05$ versus AGEs. (E) SnMP reversed the effect of anti-miR-92a-induced improvement of EDRs (F), while it did not affect SNP-induced relaxations in mouse aortas from $d b / d b$ mice. $n=3,{ }^{*} p<0.05$ versus $d b / m^{+}+$vector; ${ }^{*} p<0.05$ versus $d b / d b+$ vector; ${ }^{\S} p<0.05$ versus $d b / d b+$ anti-miR-92a. Data are mean \pm SEM. Vector, Ad-vector; anti-miR-92a, Adanti-miR-92a. (G) Schematic diagram showing miR-92a-HO-1 signaling axis in regulation of endothelial function in diabetes.

targeting miR-92a-HO-1 cascade can be an alternative therapeutic strategy for ameliorating diabetic vasculopathy.

\section{Materials and Methods}

\section{Animals}

C57BL/6 mice, $d b / d b$, and $d b / m^{+}$mice were provided by the Chinese University of Hong Kong (CUHK) Laboratory Animal Service Center and maintained at controlled temperature $\left(22-23^{\circ} \mathrm{C}, 55 \% \pm 5 \%\right.$ humidity) with a 12 -h light $/ 12$ $\mathrm{h}$ dark cycle with free access to standard mice diet (Research Diet, Inc.) and water. All animal procedures were approved by the CUHK Animal Experimentation Ethics Committee.

\section{EC culture}

HUVECs (Lonza, Walkersville, MD) were cultured in EC growth medium (CC3024; Lonza) with $10 \%$ fetal bovine serum (FBS) and antibiotics. Cells from passage 4 to 8 were used for experiments. Primary MAECs were cultured in the method of previous reports (48).

\section{Construction and production of recombinant virus}

The full human miR-92 sequences were amplified by PCR from human genomic DNA and cloned into pAAV-MCS digested by BglII and HindIII. The RGDLRVS tagged AAV (serotype 9, a gift from Dr. O.J. Müller, University Hospital Heidelberg, Heidelberg, Germany), which has higher infection efficiency in ECs and induces long-lasting expression, was used to packaging recombinant AAV (46). Empty AAVvector was used as control in corresponding experiments. Adanti-miR-92a against mature miR-92a was purchased from Vector Biolabs (USA). Empty Ad-vector was used as the control in corresponding experiments.

\section{Transfection with LNA-anti-miR-92a}

Apart from Ad-anti-miR-92a, LNA-anti-miR-92a was used to inhibit miR-92a function in HUVECs. LNA-anti- 
miR-92a and LNA-control were purchased from Exiqon Inc. with sequences as follows: 5'-AGGCCGGGACAAGTGCA AT-3' (LNA-anti-miR-92a) and 5'TAACACGTCTATAC GCCCA-3' (LNA-control). Lipofectamine ${ }^{\circledR}$ RNAiMAX Reagent (Invitrogen) was used as the transfection reagent to transfect LNA-anti-miR-92a into HUVECs. Six hours after transfection, culture medium was changed to fresh medium and HUVECs cultured for additional specified time.

\section{Construction of HO-1 3UTR reporter}

The 3'UTR of HO-1 containing the putative binding sites of miR-92a was cloned into pmirGLO (Promega) digested by Pmel and Xho1. The HO-1 3'UTR was cloned using primers as follows: forward primer: CGGGTTTAAACGGCT CCCA GGGCCATGAACTT and reverse primer: CCGTCTAGA CAAGCTACT ATCAGACAATGTT. In addition, mutated HO-1 3'UTR was cloned with a site-directed mutagenesis method. The primers were used as below:

Upper primers,

forward primer: CGGGTTTAAACGGCTCCCAGGGCC ATGAACTT and

reverse primer: ATGTCATGTTTGAGGCTGAGCCAGG Lower primers,

forward primer: AACATGACATTTTTGTTGTGTTCTG TTGTTTTTATAGC and

reverse primer: CCGTCTAGACAAGCTACTATCAGA CAATGTT

\section{Construction and production of HO-1 ShRNA AAV}

shRNA sequence targeting mouse $\mathrm{HO}-1$ was generated from Sigma-Aldrich and has been used previously (27). Briefly, the complementary oligonucleotides (oligos) of HO1 shRNA were annealed and ligated into the pAAV-ZsGreen shuttle vector. The RGDLRVS-tagged AAV (serotype 9) was used to package AAV-shHO-1 in HEK-293T cells.

\section{Organ culture of mouse aortas and functional assay}

Mouse aortas were dissected in ice-cold Krebs solution containing (in mmol/L) $119 \mathrm{NaCl}, 4.7 \mathrm{KCl}, 2.5 \mathrm{CaCl}_{2}, 1$ $\mathrm{MgCl}_{2}, 25 \mathrm{NaHCO}_{3}, 1.2 \mathrm{KH}_{2} \mathrm{PO}_{4}$, and 11 D-glucose. The thoracic aortas were cut into segments with $2 \mathrm{~mm}$ in length and cultured in Dulbecco's Modified Eagle's medium (DMEM) (low glucose, Life Technologies) supplemented with 10\% FBS (Gibco). Some aortic rings were transduced with AAV-vector and AAV-miR-92a for $36 \mathrm{~h}$ in culture medium before functional study. In addition, the chemicals, including AGEs (200 $\mu \mathrm{g} / \mathrm{ml}$; BioVision), SnMP (30 $\mu M$; Frontier Scientific), and hemin (5 $\mu M$; Sigma-Aldrich), were added individually into the culture medium as desired. The isometric force was measured by myograph (Danish Myo Technology, Aarhus, Denmark). The acetylcholine (Ach)-induced EDRs were detected after the precontraction triggered by phenylephrine (Phe) and SNP-induced EIRs were measured in the presence of LNAME $(100 \mu \mathrm{mol} / \mathrm{L}$, Fluka).

\section{ROS production in aortas and ECS}

En face staining of ROS generation using DHE fluorescence was performed in en face endothelium from mouse aortas according to the previous report (55). In brief, isolated rings from mouse aorta were incubated with DHE in extracellular medium (ECM, in mmol/L: $121 \mathrm{NaCl}, 5 \mathrm{NaHCO}_{3}, 10 \mathrm{Na}-$ HEPES, $4.7 \mathrm{KCl}, 1.2 \mathrm{KH}_{2} \mathrm{PO}_{4}, 1.2 \mathrm{MgSO}_{4}, 2 \mathrm{CaCl}_{2}, 10 \mathrm{D}-$ glucose; $\mathrm{pH}$ 7.4). After washing in ECM for three times, the aortic rings were cut open longitudinally and then placed upsidedown between two coverslips (1\#; Thermo, Braunschweig, Germany). The fluorescence images were taken on a confocal microscope (FV1000; Olympus, Tokyo, Japan) (DHE: excitation: $515 \mathrm{~nm}$, emission: $565-605 \mathrm{~nm}$; autofluorescence of elastin: excitation: $488 \mathrm{~nm}$, emission $520-535 \mathrm{~nm}$ ). Intracellular ROS were detected using DHE staining (DHE: excitation: $515 \mathrm{~nm}$, emission: 565-605 nm). Moreover, the amount of superoxide anion generation in mouse aortas was detected using the lucigenin-enhanced chemiluminescence (ECL) method (24).

\section{Western blotting}

Cells and aortic tissues were homogenized in an ice-cold RIPA lysis buffer composed of RIPA solution, complete protease inhibitor cocktail, and PhosSTOP phosphatase inhibitor cocktail (Roche). After lysis for $30 \mathrm{~min}$ on ice, the lysates were centrifuged at $20,000 \mathrm{~g}$ for $20 \mathrm{~min}$ at $4^{\circ} \mathrm{C}$. After concentration measurement, the proteins were mixed with $4 \times$ loading buffer, following by denaturation for $10 \mathrm{~min}$. Subsequently, equal amounts of protein samples were electrophoresed on a 7.5\%$12.5 \%$ sodium dodecyl sulfate/polyacrylamide gel together with the prestained protein molecular weight marker. The resolved proteins were electrophoretically transferred onto an immunoblot polyvinylidene difluoride membrane (Bio-Rad) in the Bio-Rad Trans-Blot cell at $110 \mathrm{~V}$ for $100 \mathrm{~min}$. The blots were blocked with $3 \%$ Bovine serum albumin (BSA) dissolved in TBST buffer for $1 \mathrm{~h}$, following incubation with primary antibodies, including anti-eNOS (1:1000; BD Biosciences, San Jose, CA), anti-phospho (p)-eNOS Ser1177 (1:1000; Abcam; Cambridge, United Kingdom), anti-HO-1 (1:500; Abcam), anti-NQO1 (1:1000; Abcam), anti-NOX4 (1:1000; Abcam), anti-NOX2 (1:1000; Abcam), anti-p67pox (1:500; Cell Signaling, Danvers, MA), anti-SOD1 (1:1000, Santa Cruz, California), anti-SOD2 (1:1000, Santa Cruz), and antinitrotyrosine (1:500; Millipore). Then, the membrane was washed three times in TBST and subsequently incubated with corresponding secondary antibodies conjugated to horseradish peroxidase (DakoCytomation) at the dilution of 1:3000 for $1 \mathrm{~h}$ at room temperature. After washing with TBST for three times, the membrane was developed with ECL detection solutions before exposure to X-ray films. Densitometry was performed using a documentation program (Flurochem). Glyceraldehyde 3-phosphate dehydrogenase (GAPDH) was selected as internal control for checking equal loading of each sample.

\section{En face immunofluorescence staining}

Aortic segments were fixed in $4 \%$ paraformaldehyde, followed by permeabilization with $0.01 \%$ Triton X-100 for $15 \mathrm{~min}$ at room temperature. After washing with PBS for three times, the aortic rings were blocked with 5\% normal donkey serum overnight at $4^{\circ} \mathrm{C}$, and primary antibody incubations were performed for an additional $24 \mathrm{~h}$ at $4^{\circ} \mathrm{C}$ using mouse anti-HO-1 (1:100 dilution; Abcam) and goat anti-VE cadherin (1:100 dilution; Santa Cruz) antibodies. Subsequently, the aortic segments were incubated in a corresponding fluorescent second antibody, including Alexa Fluor 488 anti-mouse and Alexa Fluor 546 anti-goat (1:500 dilution; Molecular Probes), for $2 \mathrm{~h}$ at room temperature. Finally, 
the specimens were cut open and imaged by a confocal system as described (56).

\section{Q-PCR analysis}

For mRNA detection, total RNA was extracted from ECs or mouse aortas using RNAiso plus (TAKARA) according to the manufacturer's instruction. MicroRNA detection using q-PCR analysis was performed using $\mathrm{NCode}^{\mathrm{TM}} \mathrm{SYBR}^{\circledR}$ Green miRNA q-PCR Kit (Invitrogen). The primers were purchased from GeneCopoeia (Guangzhou). U6 small nuclear RNA (snRU6) was used as endogenous control. In addition, TaqMan miRNA assay (Life Technology) was used to confirm miR-92a alterations in ABI ViiA7 system (Applied Biosystems). snRU6 was used as the internal control for miRNA detection.

\section{HO-1 activity and nitrite/nitrate assay}

Changes of HO-1 activity were measured as the bilirubin production in HUVECs. Forty-eight hours after SnMP treatment, cultured medium from HUVECs was collected for bilirubin assay. The bilirubin level was determined using an assay kit (BioVision) according to the manufacturer's instructions. NO level was measured as the accumulation of accumulated nitrite/nitrate. Briefly, NO level in cell culture medium and plasma of $d b / d b$ mice was detected using the nitrite/nitrate colorimetric assay kit (Cayman Chemicals).

\section{Luciferase assays}

To identify whether miR-92a has a potential direct regulation on HO-1 expression, we examined whether miR-92a overexpression decreased the firefly luciferase activity in HUVECs transduced with HO-1 3'UTR-containing luciferase plasmids. In brief, pAAV-miR-92a was cotransfected with WT pmirGLOHO-1 3'UTR, or mutant pmirGLO-HO-1 3'UTR using a Neon Transfection kit (Lonza). Luciferase activity was measured using a Dual-Luciferase Reporter Assay System (Promega) according to the manufacturer's protocol.

\section{Statistical analysis}

All data are presented as mean \pm standard error of mean. GraphPad Prism software (Version 5.0) was used for all data analysis. Concentration/response curves were analyzed using nonlinear regression curve fitting. Statistical significance was evaluated by Student's $t$-test or one-way ANOVA. $p<0.05$ was interpreted to denote statistical significance.

\section{Acknowledgments}

This study is supported by the Hong Kong Research Grants Council (C4024-16W, CUHK14105814 and 14124216), the Natural Science Foundation of China (81561128017), the CUHK Vice Chancellor's Discretionary Fund, and the CUHK Lui Che Woo Foundation.

\section{Author Disclosure Statement}

There are no competing financial interests.

\section{References}

1. Ahmad M, Turkseven S, Mingone CJ, Gupte SA, Wolin MS, and Abraham NG. Heme oxygenase- 1 gene expression increases vascular relaxation and decreases inducible nitric oxide synthase in diabetic rats. Cell Mol Biol 51: 371-376, 2005.

2. Barone E, Di Domenico F, Mancuso C, and Butterfield DA. The Janus face of the heme oxygenase/biliverdin reductase system in Alzheimer disease: it's time for reconciliation. Neurobiol Dis 62: 144-159, 2014.

3. Bartel DP. MicroRNAs: target recognition and regulatory functions. Cell 136: 215-233, 2009.

4. Basta G, Schmidt AM, and De Caterina R. Advanced glycation end products and vascular inflammation: implications for accelerated atherosclerosis in diabetes. Cardiovasc Res 63: 582-592, 2004.

5. Bonauer A, Carmona G, Iwasaki M, Mione M, Koyanagi M, Fischer A, Burchfield J, Fox H, Doebele C, Ohtani K, Chavakis E, Potente M, Tjwa M, Urbich C, Zeiher AM, and Dimmeler S. MicroRNA-92a controls angiogenesis and functional recovery of ischemic tissues in mice. Science 324: 1710-1713, 2009.

6. Chen YH, Lin SJ, Lin MW, Tsai HL, Kuo SS, Chen JW, Charng MJ, Wu TC, Chen LC, Ding PYA, Pan WH, Jou YS, and Chau LY. Microsatellite polymorphism in promoter of heme oxygenase-1 gene is associated with susceptibility to coronary artery disease in type 2 diabetic patients. Hum Genet 111: 1-8, 2002.

7. Chen Z, Wen L, Martin M, Hsu CY, Fang L, Lin FM, Lin TY, Geary MJ, Geary GG, Zhao Y, Johnson DA, Chen JW, Lin SJ, Chien S, Huang HD, Miller YI, Huang PH, and Shyy JY. Oxidative stress activates endothelial innate immunity via sterol regulatory element binding protein 2 (SREBP2) transactivation of microRNA-92a. Circulation 131: 805-814, 2015.

8. Cheng X, Siow RC, and Mann GE. Impaired redox signaling and antioxidant gene expression in endothelial cells in diabetes: a role for mitochondria and the nuclear factorE2-related factor 2-Kelch-like ECH-associated protein 1 defense pathway. Antioxid Redox Signal 14: 469-487, 2011.

9. Condorelli G, Latronico MV, and Cavarretta E. microRNAs in cardiovascular diseases: current knowledge and the road ahead. J Am Coll Cardiol 63: 2177-2187, 2014.

10. Csonka C, Varga E, Kovacs P, Ferdinandy P, Blasig IE, Szilvassy Z, and Tosaki A. Heme oxygenase and cardiac function in ischemic/reperfused rat hearts. Free Radic Biol Med 27: 119-126, 1999.

11. Daniel JM, Penzkofer D, Teske R, Dutzmann J, Koch A, Bielenberg W, Bonauer A, Boon RA, Fischer A, Bauersachs J, van Rooij E, Dimmeler S, and Sedding DG. Inhibition of miR-92a improves re-endothelialization and prevents neointima formation following vascular injury. Cardiovasc Res 103: 564-572, 2014.

12. Doebele C, Bonauer A, Fischer A, Scholz A, Reiss Y, Urbich C, Hofmann WK, Zeiher AM, and Dimmeler S. Members of the microRNA-17-92 cluster exhibit a cellintrinsic antiangiogenic function in endothelial cells. Blood 115: 4944-4950, 2010.

13. El-Bassossy HM, El-Maraghy NN, El-Fayoumi HM, and Watson ML. Haem oxygenase-1 induction protects against tumour necrosis factor alpha impairment of endothelialdependent relaxation in rat isolated pulmonary artery. $\mathrm{Br} \mathrm{J}$ Pharmacol 158: 1527-1535, 2009.

14. Fang Y and Davies PF. Site-specific microRNA-92a regulation of Kruppel-like factors 4 and 2 in atherosusceptible endothelium. Arterioscler Thromb Vasc Biol 32: 979-987, 2012 . 
15. Fledderus JO, Boon RA, Volger OL, Hurttila H, YlaHerttuala S, Pannekoek H, Levonen AL, and Horrevoets AJG. KLF2 primes the antioxidant transcription factor Nrf2 for activation in endothelial cells. Arterioscler Thromb Vasc Biol 28: 1339-1346, 2008.

16. Galle J, Heinloth A, Wanner C, and Heermeier K. Dual effect of oxidized LDL on cell cycle in human endothelial cells through oxidative stress. Kidney Int Suppl 59: S120S123, 2001.

17. Giacco F and Brownlee M. Oxidative stress and diabetic complications. Circ Res 107: 1058-1070, 2010.

18. Goldin A, Beckman JA, Schmidt AM, and Creager MA. Advanced glycation end products-sparking the development of diabetic vascular injury. Circulation 114: 597-605, 2006.

19. Haines DD, Lekli I, Teissier P, Bak I, and Tosaki A. Role of haeme oxygenase-1 in resolution of oxidative stress-related pathologies: focus on cardiovascular, lung, neurological and kidney disorders. Acta Physiologica 204: 487-501, 2012.

20. Inzucchi SE and Amatruda JM. Lipid management in patients with diabetes: translating guidelines into action. Diabetes Care 26: 1309-1311, 2003.

21. Kang YS, Lee MH, Song HK, Hyun YY, Cha JJ, Ko GJ, Kim SH, Lee JE, Han JY, and Cha DR. Aliskiren improves insulin resistance and ameliorates diabetic vascular complications in $\mathrm{db} / \mathrm{db}$ mice. Nephrol Dial Transplant 26: 1194-1204, 2011.

22. Kruger AL, Peterson S, Turkseven S, Kaminski PM, Zhang FF, Quan S, Wolin MS, and Abraham NG. D-4F induces heme oxygenase-1 and extracellular superoxide dismutase, decreases endothelial cell sloughing, and improves vascular reactivity in rat model of diabetes. Circulation 111: 31263134, 2005.

23. Laight DW, Carrier MJ, and Anggard EE. Antioxidants, diabetes and endothelial dysfunction. Cardiovasc Res 47: 457-464, 2000.

24. Lau YS, Tian XY, Mustafa MR, Murugan D, Liu J, Zhang Y, Lau CW, and Huang Y. Boldine improves endothelial function in diabetic $\mathrm{db} / \mathrm{db}$ mice through inhibition of angiotensin II-mediated BMP4-oxidative stress cascade. $\mathrm{Br} \mathrm{J}$ Pharmacol 170: 1190-1198, 2013.

25. Li J, Li JF, Wei TT, and Li JH. Down-regulation of microRNA-137 improves high glucose-induced oxidative stress injury in human umbilical vein endothelial cells by up-regulation of AMPK alpha 1. Cell Physiol Biochem 39: 847-859, 2016.

26. Lin R, Fu WQ, Zhou WC, Wang Y, Wang XF, Huang W, and Jin L. Association of heme oxygenase-1 gene polymorphisms with essential hypertension and blood pressure in the Chinese Han population. Genet Test Mol Biomarkers 15: 23-28, 2011.

27. Liu J, Wang L, Tian XY, Liu L, Wong WT, Zhang Y, Han QB, Ho HM, Wang N, Wong SL, Chen ZY, Yu J, Ng CF, Yao $\mathrm{X}$, and Huang $\mathrm{Y}$. Unconjugated bilirubin mediates heme oxygenase-1-induced vascular benefits in diabetic mice. Diabetes 64: 1564-1575, 2015.

28. Loyer X, Potteaux S, Vion AC, Guerin CL, Boulkroun S, Rautou PE, Ramkhelawon B, Esposito B, Dalloz M, Paul JL, Julia P, Maccario J, Boulanger CM, Mallat Z, and Tedgui A. Inhibition of microRNA-92a prevents endothelial dysfunction and atherosclerosis in mice. Circ Res 114: 434-443, 2014.
29. Magenta A, Carlomosti F, D'agostino M, Beji S, Zaccagnini G, Maimone B, Di Stefano V, De Santa F, Cordisco S, Antonini A, Ciarapica R, Dellambra E, Martelli F, Avitabile D, and Capogrossi MC. Oxidative stressinduced miR-200c disrupts the regulatory loop among SIRT1, FOXO1 and eNOS. Antioxid Redox Signal PMID: 27960536, 2016.

30. Martinez-Hernandez A, Cordova EJ, Rosillo-Salazar O, Garcia-Ortiz H, Contreras-Cubas C, Islas-Andrade S, RevillaMonsalve C, Salas-Labadia C, and Orozco L. Association of HMOX1 and NQO1 polymorphisms with metabolic syndrome components. PLoS One 10: e0123313, 2015.

31. Montero D, Walther G, Stehouwer CD, Houben AJ, Beckman JA, and Vinet A. Effect of antioxidant vitamin supplementation on endothelial function in type 2 diabetes mellitus: a systematic review and meta-analysis of randomized controlled trials. Obes Rev 15: 107-116, 2014.

32. Nascimento NR, Lessa LM, Kerntopf MR, Sousa CM, Alves RS, Queiroz MG, Price J, Heimark DB, Larner J, Du X, Brownlee M, Gow A, Davis C, and Fonteles MC. Inositols prevent and reverse endothelial dysfunction in diabetic rat and rabbit vasculature metabolically and by scavenging superoxide. Proc Natl Acad Sci U S A 103: 218-223, 2006.

33. Ono K, Kuwabara Y, and Han J. MicroRNAs and cardiovascular diseases. FEBS J 278: 1619-1633, 2011.

34. Paneni F, Beckman JA, Creager MA, and Cosentino F. Diabetes and vascular disease: pathophysiology, clinical consequences, and medical therapy: part I. Eur Heart $J$ 34: 2436-2443, 2013.

35. Pataki T, Bak I, Csonka C, Kovacs P, Varga E, Blasig IE, and Tosaki A. Regulation of ventricular fibrillation by heme oxygenase in ischemic/reperfused hearts. Antioxid Redox Signal 3: 125-134, 2001.

36. Potenza MA, Gagliardi S, Nacci C, Carratu MR, and Montagnani M. Endothelial dysfunction in diabetes: from mechanisms to therapeutic targets. Curr Med Chem 16: 94 112, 2009.

37. Pricci F, Leto G, Amadio L, Iacobini C, Cordone S, Catalano S, Zicari A, Sorcini M, Di Mario UR, and Pugliese G. Oxidative stress in diabetes-induced endothelial dysfunction involvement of nitric oxide and protein kinase C. Free Radic Biol Med 35: 683-694, 2003.

38. Ryter SW and Choi AMK. Heme oxygenase-1/carbon monoxide from metabolism to molecular therapy. Am J Respir Cell Mol Biol 41: 251-260, 2009.

39. Sena CM, Nunes E, Louro T, Proenca T, and Seica RM. Endothelial dysfunction in type 2 diabetes: effect of antioxidants. Rev Port Cardiol 26: 609-619, 2007.

40. Sharp FR, Zhan XH, and Liu DZ. Heat shock proteins in the brain: role of Hsp70, Hsp 27, and HO-1 (Hsp32) and their therapeutic potential. Transl Stroke Res 4: 685-692, 2013.

41. Tabit CE, Chung WB, Hamburg NM, and Vita JA. Endothelial dysfunction in diabetes mellitus: molecular mechanisms and clinical implications. Rev Endocr Metab Disord 11: 61-74, 2010.

42. Tan KC, Chow WS, Ai VH, Metz C, Bucala R, and Lam KS. Advanced glycation end products and endothelial dysfunction in type 2 diabetes. Diabetes Care 25: 10551059, 2002.

43. Tian XY, Wong WT, Xu A, Lu Y, Zhang Y, Wang L, Cheang WS, Wang Y, Yao X, and Huang Y. Uncoupling 
protein-2 protects endothelial function in diet-induced obese mice. Circ Res 110: 1211-1216, 2012.

44. Tunceli K, Pladevall M, Williams LK, Divine GW, Simpkins JC, Nag SS, Sajjan SG, Kamal-Bahl SJ, Alexander $\mathrm{CM}$, and Lafata JE. Trends in lipid management among patients with diabetes. Endocr Pract 12: 380-387, 2006.

45. Turkseven S, Kruger A, Mingone CJ, Kaminski P, Inaba M, Rodella LF, Ikehara S, Wolin MS, and Abraham NG. Antioxidant mechanism of heme oxygenase-1 involves an increase in superoxide dismutase and catalase in experimental diabetes. Am J Physiol Heart Circ Physiol 289: H701-H707, 2005.

46. Varadi K, Michelfelder S, Korff T, Hecker M, Trepel M, Katus HA, Kleinschmidt JA, and Muller OJ. Novel random peptide libraries displayed on AAV serotype 9 for selection of endothelial cell-directed gene transfer vectors. Gene Ther 19: 800-809, 2012.

47. Watts GF, Playford DA, Croft KD, Ward NC, Mori TA, and Burke V. Coenzyme Q(10) improves endothelial dysfunction of the brachial artery in Type II diabetes mellitus. Diabetologia 45: 420-426, 2002.

48. Wong WT, Tian XY, Xu AM, Yu J, Lau CW, Hoo RLC, Wang Y, Lee VWY, Lam KSL, Vanhoutte PM, and Huang Y. Adiponectin is required for PPAR gamma-mediated improvement of endothelial function in diabetic mice. Cell Metab 14: 104-115, 2011.

49. Wu C, Huang RT, Kuo CH, Kumar S, Kim CW, Lin YC, Chen YJ, Birukova A, Birukov KG, Dulin NO, Civelek M, Lusis AJ, Loyer X, Tedgui A, Dai G, Jo H, and Fang Y. Mechanosensitive PPAP2B regulates endothelial responses to atherorelevant hemodynamic forces. Circ Res 117: e41e53, 2015.

50. Wu W, Xiao H, Laguna-Fernandez A, Villarreal G, Wang KC, Geary GG, Zhang YZ, Wang WC, Huang HD, Zhou J, Li YS, Chien S, Garcia-Cardena G, and Shyy JYJ. Flowdependent regulation of Kruppel-like factor 2 is mediated by microRNA-92a. Circulation 124: 633-641, 2011.

51. Xie Y and Chen Y. microRNAs: emerging targets regulating oxidative stress in the models of Parkinson's disease. Front Neurosci 10: 298, 2016.

52. Yamada J, Yoshimura S, Yamakawa H, Sawada M, Nakagawa M, Hara S, Kaku Y, Iwama T, Naganawa T, Banno Y, Nakashima S, and Sakai N. Cell permeable ROS scavengers, Tiron and Tempol, rescue PC12 cell death caused by pyrogallol or hypoxia/reoxygenation. Neurosci Res 45: 1-8, 2003.

53. Yu M, Liu Y, Zhang B, Shi Y, Cui L, and Zhao X. Inhibiting microRNA-144 abates oxidative stress and reduces apoptosis in hearts of streptozotocin-induced diabetic mice. Cardiovasc Pathol 24: 375-381, 2015.

54. Zhang HN, Liu J, Qu D, Wang L, Luo JY, Lau CW, Liu PS, Gao Z, Tipoe GL, Lee HK, Ng CF, Ma RCW, Yao XQ, and Huang Y. Inhibition of miR-200c restores endothelial function in diabetic mice through suppression of COX-2. Diabetes 65: 1196-1207, 2016.

55. Zhang Y, Liu J, Luo JY, Tian XY, Cheang WS, Xu J, Lau CW, Wang L, Wong WT, Wong CM, Lan HY, Yao $\mathrm{XQ}$, Raizada MK, and Huang Y. Upregulation of angiotensin (1-7)-mediated signaling preserves endothelial function through reducing oxidative stress in diabetes. Anioxid Redox Signal 23: 880-892, 2015.

56. Zhang Y, Liu J, Tian XY, Wong WT, Chen YC, Wang L, Luo JY, Cheang WS, Lau CW, Kwan KM, Wang NP, Yao $\mathrm{XQ}$, and Huang $\mathrm{Y}$. Inhibition of bone morphogenic protein 4 restores endothelial function in $\mathrm{db} / \mathrm{db}$ diabetic mice. $A r$ terioscler Thromb Vasc Biol 34: 152-159, 2014.
Address correspondence to:

Dr. Yu Huang

School of Biomedical Sciences

Chinese University of Hong Kong

Room 222

Lo Kwee-Seong Integrated

Biomedical Sciences Building

Hong Kong 00852

China

E-mail: yu-huang@cuhk.edu.hk

Dr. Jiang-Yun Luo

School of Biomedical Sciences

Chinese University of Hong Kong

Room 222

Lo Kwee-Seong Integrated

Biomedical Sciences Building

Hong Kong 00852

China

E-mail: luojiangyun1985@gmail.com

Date of first submission to ARS Central, January 27, 2017; date of final revised submission, June 8, 2017; date of acceptance, June 26, 2017.

\begin{tabular}{|c|}
\hline $\begin{aligned} & \text { Abbreviations Used } \\
\text { AAV } & =\text { adeno-associated virus } \\
\text { ACh } & =\text { acetylcholine } \\
\text { AGEs } & =\text { advanced glycation end products } \\
\text { CUHK } & =\text { Chinese University of Hong Kong } \\
\text { DHE } & =\text { dihydroethidium } \\
\mathrm{ECL} & =\text { enhanced chemiluminescence } \\
\mathrm{ECM} & =\text { extracellular medium } \\
\mathrm{ECs} & =\text { endothelial cells } \\
\mathrm{EDR} & =\text { endothelium-dependent relaxation } \\
\mathrm{eNOS} & =\text { endothelial nitric oxide synthases } \\
\mathrm{FBS} & =\text { fetal bovine serum } \\
\mathrm{HO}-1 & =\text { heme oxygenase-1 } \\
\mathrm{HUVEC} & =\text { human umbilical vein endothelial cells } \\
\mathrm{HX}-\mathrm{XO} & =100 \mu \text { mol/L hypoxanthine }+0.01 \text { units } / \mathrm{mL} \\
\mathrm{KLF} 2 & =\text { krüppel-like factor } 2 \\
\mathrm{MAECs} & =\text { mouse aortic endothelial cells } \\
\text { miR-92a } & =\text { microRNA-92a } \\
\text { miRNAs } & =\text { microRNAs } \\
\mathrm{NF}-\kappa \mathrm{B} & =\text { nuclear factor } \kappa \mathrm{B} \\
\mathrm{NO} & =\text { nitric oxide } \\
\text { oxLDL } & =\text { oxidized low-density lipoprotein } \\
\mathrm{PCR} & =\text { polymerase chain reaction } \\
\mathrm{Phe} & =\text { phenylephrine } \\
\mathrm{q}-\mathrm{PCR} & =\text { quantitative polymerase chain reaction } \\
\mathrm{ROS} & =\text { reactive oxygen species } \\
\mathrm{SEM} & =\text { standard error of mean } \\
\mathrm{SnMP} & =\text { tin mesoporphyrin } \\
\mathrm{SNP} & =\text { sodium nitroprusside } \\
\mathrm{snRU6} & =\text { U6 small nuclear RNA } \\
\mathrm{SREBP} 2 & =\text { sterol regulatory element binding protein } 2 \\
\mathrm{WT} & =\text { wild type }\end{aligned}$ \\
\hline
\end{tabular}

